\title{
Approaches to Multimodality Imaging of Angiogenesis
}

Lawrence W. Dobrucki ${ }^{1}$, Ebo D. de Muinck ${ }^{2}$, Jonathan R. Lindner ${ }^{3}$, and Albert J. Sinusas ${ }^{1}$

${ }^{1}$ School of Medicine, Yale University, New Haven, Connecticut; ${ }^{2}$ Linköping University, Linköping, Sweden; and ${ }^{3}$ Oregon Health and Science University, Portland, Oregon

Angiogenesis, defined as the formation of new capillaries by cellular outgrowth from existing microvessels, can be assessed by the evaluation of perfusion, function, and metabolism. However, more recently, novel, noninvasive imaging strategies for the evaluation of molecular events associated with the angiogenic process have been developed.

J Nucl Med 2010; 51:66S-79S

DOI: 10.2967/jnumed.110.074963

$V_{\text {artosul }}$ arious imaging modalities and technologies for mapping biomolecular or biologic processes within single cells or even whole organs have the extraordinary potential to revolutionize the diagnosis and treatment of pathophysiologic disorders and, in turn, to mitigate the significant social and economic costs associated with the clinical management of diseases. Such integrated imaging approaches will eventually lead to individualized programs for disease prevention through advanced diagnosis, risk stratification, and targeted cell therapies, resulting in more successful and efficient health care. The goal of this article is to provide readers with a current update of selected state-of-the-art imaging modalities and quantitative imaging strategies that might lead to improved clinical outcomes; these include targeted nuclear, ultrasound, and MRI approaches for assessing naturally occurring and therapeutic angiogenesis.

\section{ANGIOGENESIS}

Angiogenesis represents the sprouting of new capillaries from existing microvasculature. Angiogenesis is a multistep process orchestrated by a host of angiogenic factors and inhibitors, which offer a wide range of targets for therapeutic interventions and imaging (1). During the past decade, intensive research has been focused on promoting revascularization in ischemic tissues by stimulating angiogenesis and arteriogenesis, the remodeling of preexisting arteries or noncapillary microvessels. Preclinical studies

Received Jan. 12, 2010; revision accepted Feb. 16, 2010.

For correspondence or reprints contact: Lawrence W. Dobrucki, Department of Internal Medicine, School of Medicine, Yale University,

P.O. Box 208017, New Haven, CT 06513.

E-mail: wawrzyniec.dobrucki@yale.edu

COPYRIGHT () 2010 by the Society of Nuclear Medicine, Inc. have demonstrated the benefit of administering growth factors, such as vascular endothelial growth factor (VEGF) or fibroblast growth factor (FGF), to promote angiogenesis in models of ischemic injury (2). Unfortunately, randomized clinical trials with recombinant or adenoviral VEGF or FGF delivered locally did not demonstrate a clear benefit in patients with peripheral artery disease or coronary artery disease (3). Part of this failure was attributable to suboptimal delivery strategies, the use of a single growth factor, lack of knowledge of optimal doses and durations of therapies, and the formation of nonfunctional vessels. Therefore, novel strategies involving transplantation of stem cells, delivery of nanoparticles capable of releasing multiple growth factors at various times during vascular development, and stimulation of the growth of proximal collateral conduits may be required. The evaluation of therapeutic angiogenesis in these trials focused on several clinical endpoints, including symptoms, exercise tolerance, measures of quality of life and survival, pressure measurements in peripheral artery disease, and imaging of tissue perfusion in coronary artery disease. To address the need to monitor novel cell-based or genetic therapies, the development of noninvasive approaches for the direct evaluation of molecular events associated with angiogenesis is required (3).

\section{CHOICE OF IMAGING TECHNOLOGY AND PROBES}

The development of noninvasive approaches for monitoring angiogenesis is progressing concurrently in 2 directions; one is the advancement of current imaging technology, and the other is the identification of novel targets with biosignatures unique to the angiogenic process. Although numerous imaging modalities have been developed during recent decades, only a few are available for broad application in both preclinical and clinical molecular imaging of biologic processes. Table 1 shows selected operational parameters of various clinical imaging modalities and their advantages and disadvantages for the targeted molecular imaging of angiogenesis. These characteristics are based on underlying physical and chemical differences; therefore, the selection of an imaging modality should be based on the accessibility of instrumentation, the availability and performance of targeted probes, and the properties of the biologic system to be studied. 


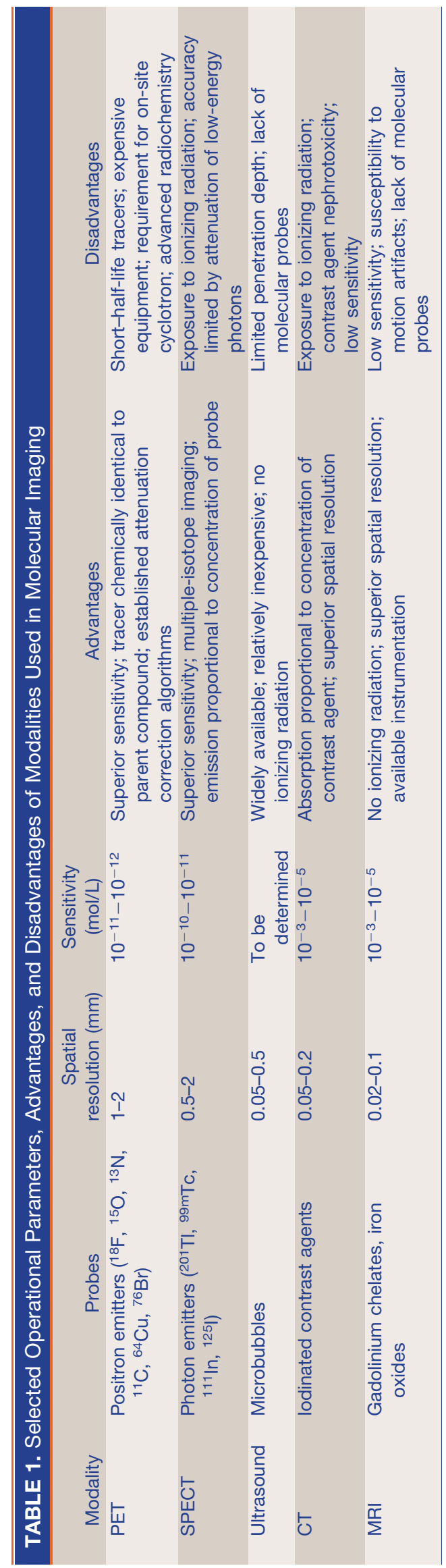

Despite the evolution of imaging technology, characterized by the development of high-resolution, high-sensitivity imaging systems and, more recently, dedicated hybrid imaging systems (which combine 2 or more imaging modalities), the identification of unique biologic targets and the creation of targeted imaging probes are equally important in the advancement of molecular imaging. Potential novel biologic targets for the imaging of angiogenesis are defined elsewhere in this supplement to The Journal of Nuclear Medicine. Targets for the imaging of angiogenesis have traditionally been divided into 3 major categories: nonendothelial cell targets, such as molecules associated with proangiogenic monocytes, macrophages, and stem cells; endothelial cell targets, such as VEGF, growth factor receptors, integrins, CD13, cell adhesion molecules (such as vascular cell adhesion molecule 1 [VCAM-1]), and syndecan 4; and extracellular matrix proteins and matrix proteases (4). Among the potential targets, VEGF, VEGF receptors, and integrins, in particular, have been identified as the most favorable targets for the imaging of angiogenesis; therefore, attention has been directed to the development of molecular probes for these specific targets.

The discovery of the RGD (arginine-glycine-aspartate) sequence as the main binding motif for integrins was the starting point for the rapid development of probes targeted at $\alpha_{v} \beta_{3}$-integrin and having suitable in vivo characteristics for the molecular imaging of angiogenesis. First-generation linear peptides had low selectivity and stability, whereas agents based on antibodies had high selectivity but had long blood residence times and high background activity. During the past decade, a variety of $\alpha_{\mathrm{v}} \beta_{3}$-integrin-targeted lead constructs, such as the cyclic RGD core, evolved, allowing for the optimization of pharmacokinetics (via the attachment of carbohydrates, charged amino acids, and polyethylene glycol analogs) and binding characteristics (via multimerization). More recently, the use of both organic and inorganic nanoparticles, such as nanotubes, quantum dots, or paramagnetic particles, has gained interest. The surface of nanoparticles can be functionalized so that a signaling moiety (such as a radioisotope, fluorophore, or paramagnetic molecule), targeting ligands (such as cyclic RGD analogs), and polyethylene glycol can be attached to provide a detectable signal, target the particles, and alter the pharmacokinetics of the particles, respectively. Moreover, nanoparticles have the ability to deliver several different types of imaging agents so that multimodality imaging can be performed, and their large surface area promises the achievement of high sensitivity. Table 2 shows selected targeted probes for the molecular imaging of angiogenesis with modalities that are described in more detail in later sections.

\section{NUCLEAR APPROACHES}

SPECT and PET have been the main modalities used for physiologic imaging in patients and molecular imaging of biochemical processes. Because of their high sensitivity (in the picomolar range), their reasonable spatial resolution (in 
TABLE 2. Selected Targeted Probes for Molecular Imaging of Angiogenesis

\begin{tabular}{|c|c|c|c|c|}
\hline Marker & Probe & Modality & Biologic target or application & Reference \\
\hline \multirow[t]{8}{*}{ VEGF } & 124I-VG76e & PET & Tumor angiogenesis & Collingridge et al. (5) \\
\hline & ${ }^{123}$ I-VEGF 165 & SPECT & Tumor angiogenesis & Li et al. (6) \\
\hline & ${ }^{111}$ In-VEGF 121 & SPECT & Peripheral limb angiogenesis & Lu et al. (63) \\
\hline & ${ }^{64} \mathrm{Cu}-\mathrm{DOTA}-\mathrm{VEGF}_{121}$ & PET & Tumor angiogenesis & Cai et al. (7) \\
\hline & ${ }^{64} \mathrm{Cu}-\mathrm{DOTA}-\mathrm{VEGF}_{121}$ & PET & Myocardial angiogenesis & Rodriquez-Porcel et al. (8) \\
\hline & 99mTc-scVEGF & SPECT & Peripheral limb angiogenesis & Levashova et al. (10) \\
\hline & ${ }^{64} \mathrm{Cu}$-scVEGF & PET & Tumor angiogenesis & Backer et al. (9) \\
\hline & Anti-VEGFR2 antibody & Ultrasound & Tumor angiogenesis & Willmann et al. (40) \\
\hline \multirow[t]{18}{*}{$\alpha_{\mathrm{v}} \beta_{3}$} & ${ }^{111} \ln -\mathrm{RP} 748$ & SPECT & Tumor angiogenesis & Harris et al. (14) \\
\hline & & & Myocardial angiogenesis & Meoli et al. (16) \\
\hline & ${ }^{18} \mathrm{~F}-\mathrm{AH} 111585$ & PET & Tumor angiogenesis & Kenny et al. (64), Morrison et al. (65) \\
\hline & 123|-RGD & SPECT & Myocardial angiogenesis & Johnson et al. (66) \\
\hline & ${ }^{125}$ I-c(RGD(l)yV) & SPECT & Peripheral limb angiogenesis & Lee et al. (67) \\
\hline & 99mTc-NC100692 & SPECT & Peripheral limb angiogenesis & Hua et al. (21) \\
\hline & & & Myocardial angiogenesis & Lindsey et al. (23) \\
\hline & ${ }^{18} \mathrm{~F}-\mathrm{NC} 100717$ & PET & Angiogenic tumor vasculature & Indrevoll et al. (68) \\
\hline & ${ }^{11} \mathrm{C}-\mathrm{NC} 100717$ & PET & Angiogenic tumor vasculature & Indrevoll et al. (68) \\
\hline & ${ }^{76} \mathrm{Br}$-nanoparticles & PET & Peripheral limb angiogenesis & Almutairi et al. (30) \\
\hline & Echistatin microbubbles & Ultrasound & Tumor angiogenesis & Ellegala et al. (36) \\
\hline & & & Peripheral angiogenesis & Leong-Poi et al. $(37,38)$ \\
\hline & RRL microbubbles & Ultrasound & Tumor angiogenesis & Weller et al. (39) \\
\hline & LM609 antibody & MRI & Tumor angiogenesis & Sipkins et al. (12) \\
\hline & RGD-liposomal nanoparticles & MRI & Angiogenesis & Kluza et al. (48) \\
\hline & RGD-SPIO nanoparticles & MRI & Tumor angiogenesis & Zhang et al. (50) \\
\hline & $\begin{array}{l}\text { RGD-cross-linked iron } \\
\text { oxide-Cy } 5.5\end{array}$ & MRI & Tumor angiogenesis & Montet et al. (51) \\
\hline & $\begin{array}{l}\text { CD13/aminopeptidase } \mathrm{N} \\
\text { quantum dots }\end{array}$ & MRI & Tumor angiogenesis & Oostendorp et al. (57) \\
\hline
\end{tabular}

scVEGF $=$ single-chain VEGF .

the millimeter range), and the availability of targeted probes and instrumentation, SPECT and PET are well suited for the molecular imaging of angiogenesis.

Early studies involved the use of radiolabeled monoclonal antibodies specific for the epitopes expressed during the formation of neovasculature to monitor the progression of cancer-associated angiogenesis. Collingridge et al. (5) and Li et al. (6) used human monoclonal anti-VEGF antibodies labeled with radioiodine ${ }^{124} \mathrm{I}$ and ${ }^{123}$ I) for PET imaging and SPECT imaging, respectively. Despite the high selectivity achieved with the use of antibodies, their slow clearance rate limited the use of this approach to preclinical studies. Alternative probes designed to overcome this limitation were identified by in vivo screening of phage display peptide libraries. This approach resulted in the development of radiotracers for monitoring angiogenesis with both the VEGF receptors that are upregulated in the remodeling microcirculation and the ligands as the targets. Initial experiments were carried out with iodinated VEGF isoforms VEGF $_{165}$ and VEGF $_{121}$ to assess tumor angiogenesis. Radiolabeling with ${ }^{123} \mathrm{I}$ did not influence the functional properties of either of the proteins, and both radiotracers were used successfully in patients (6). The $\mathrm{VEGF}_{121}$ isoform was labeled with ${ }^{111}$ In and used as a targeting ligand to assess peripheral angiogenesis in rabbits with SPECT or was labeled with ${ }^{64} \mathrm{Cu}$ for PET of mouse tumors (7) and infarcted myocardium in rats (8).

In another strategy for the imaging of VEGF receptors in angiogenic vasculature, a fusion protein containing 2 fragments of human $\mathrm{VEGF}_{121}$ cloned head to tail was used. This single-chain VEGF was labeled with the ${ }^{99 \mathrm{~m}} \mathrm{Tc}$ chelator hydraziniumpyridine for SPECT and with the ${ }^{64} \mathrm{Cu}$ chelator polyethylene glycol-1,4,7,10-tetraazocyclododecane- $N, N^{\prime}, N^{\prime \prime}, N^{\prime \prime \prime}$-tetraacetic acid (DOTA) for PET of tumor-associated angiogenic vasculature $(9,10)$.

The noninvasive identification of VEGF receptors could provide a tool for identifying sites for the local delivery of angiogenic factors, therapeutic gene vectors, and stem cells to promote an angiogenic response in ischemic tissues. This type of reporter gene technology was used to detect the expression of $\mathrm{VEGF}_{121}$ with PET/CT in porcine myocardium after adenoviral transfer (11). In that study, the authors demonstrated the value of integrated multimodal systems for detecting VEGF overexpression (with PET) and for localizing uptake to the myocardium (with CT). Moreover, VEGF overexpression after adenoviral transfer increased myocardial perfusion without significant upregulation of $\alpha_{v} \beta_{3}$-integrin.

$\alpha_{v} \beta_{3}$-integrin is the most abundant integrin expressed on the surface of proliferating endothelial cells and has been implicated in cell migration and cell survival signaling. 
These properties have made $\alpha_{\mathrm{v}} \beta_{3}$-integrin a target of choice for the imaging of angiogenesis. Initial in vivo evaluations were performed in a rabbit model of squamous cell carcinoma with a biotinylated monoclonal antibody (LM609) against $\alpha_{v} \beta_{3}$-integrin (12). Despite the high selectivity of the antibody, the slow in vivo clearance rate of antibodies has hindered their use; therefore, other research groups have focused on the development of novel imaging agents by screening phage display libraries to identify potential candidates with improved tracer kinetics, a favorable biodistribution, and high selectivity. The RGD tripeptide sequence, naturally present in extracellular matrix proteins, was found to be highly selective for $\alpha_{v} \beta_{3}$-integrin. The first in vivo evaluations focused on a potent inhibitor of $\alpha_{v} \beta_{3^{-}}$ integrin containing the pentapeptide sequence Arg-GlyAsp-D-Tyr-Val (cRGDfV). Although the initial constructs were lipophilic and were cleared via the hepatobiliary route, resulting in high liver uptake, several other compounds were synthesized with the aim of improving pharmacokinetics and clearance through the kidneys. These cyclic monomeric agents were labeled with ${ }^{125} \mathrm{I}$ and ${ }^{18} \mathrm{~F}$ and were used successfully for SPECT and PET of tumor angiogenesis (13).

Besides radiometalated RGD peptides, radiolabeled small-molecule peptidomimetic agents have been used by other groups. The high affinity and selectivity of a ${ }^{111} \mathrm{In}-$ labeled quinolone ( ${ }^{111} \mathrm{In}$-RP748) for activated $\alpha_{v} \beta_{3}$-integrin were reported in assays of integrin-mediated adhesion (14) and confirmed in an animal model of vascular injury (15). Meoli et al. evaluated the uptake and clearance of ${ }^{111} \mathrm{In}-$ RP748 in rodents and canines at early and late times after myocardial infarction in relation to perfusion, tissue hypoxia, and histochemical markers of angiogenesis (16). They observed increased uptake of ${ }^{111} \mathrm{In}-\mathrm{RP748}$ in the infarcted regions in association with the activation of $\alpha_{v} \beta_{3}$-integrin and reduced myocardial perfusion assessed with ${ }^{99 \mathrm{~m}} \mathrm{Tc}-$ sestamibi (16). Dual-isotope imaging with ${ }^{111}$ In-RP748 and radiolabeled nitroimidazole $\left({ }^{99 \mathrm{~m}} \mathrm{Tc}-\mathrm{BRU} 5921\right)$ was also used to assess myocardial angiogenesis early after infarction (17). The regional retention of ${ }^{111}$ In-RP748 in the reperfused infarcted regions correlated well with the uptake of ${ }^{99 \mathrm{~m}} \mathrm{Tc}-\mathrm{BRU} 5921$, which was taken up by hypoxic myocardium (17).

Although most initial cyclic RGD peptide agents were monomeric, several groups found that multimeric RGD peptides (dimers, tetramers, and octamers) had much higher integrin affinity, presumably because multivalent interactions with target cells resulted in improved targeting efficacy relative to that of monomeric RGD analogs (18-20).

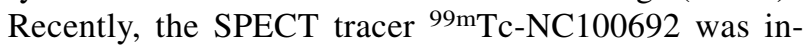
troduced for the imaging of $\alpha_{\mathrm{v}} \beta_{3}$-integrin expression in various preclinical animal models of tumor and ischemiainduced angiogenesis. Hua et al. evaluated ${ }^{99 \mathrm{~m} T c-N C 100692}$ for the planar imaging of angiogenesis in an established murine model of hind-limb ischemia (21). Increased focal ${ }^{99 m}$ Tc-NC100692 activity was seen distal to the femoral artery occlusion at days 3 and 7 and was correlated with immunohistochemical indices of angiogenesis. The specificity and localization of the targeted tracer were confirmed by dual immunofluorescence staining of endothelial cell marker CD31 and a fluorescent analog of ${ }^{99 \mathrm{~m}} \mathrm{Tc}-\mathrm{NC} 100692$ (21). That initial study demonstrated a positive linear correlation between quantitative uptake derived from planar images of 99m Tc-NC100692 and $\gamma$-well counting of tissue sections; however, the analysis of in vivo planar images tended to underestimate the magnitude of the relative uptake of ${ }^{99 \mathrm{~m} T c-}$ NC100692 in the ischemic hind limbs. This underestimation of tissue activity was attributed to errors in the selection of regions of interest or attenuation and partial-volume errors.

Subsequent studies by Dobrucki et al., using highresolution hybrid micro-SPECT/CT, provided a more accurate estimation of relative and absolute ${ }^{99 \mathrm{~m} T c-N C 100692}$ uptake in the ischemic hind limbs (22). These investigators validated the reproducibility, accuracy, and applicability of a semiautomated noninvasive approach for the serial quantitative evaluation of targeted micro-SPECT/CT images of peripheral angiogenesis in mice. In that study, the lower-resolution, high-sensitivity micro-SPECT images were coregistered with the high-resolution anatomic micro$\mathrm{CT}$ images for quantitative analysis. CT-based segmentation of the murine hind limbs defined 3-dimensional anatomic regions of interest that were applied to the micro-SPECT images for the quantification of radiotracer uptake. This quantitative hybrid micro-SPECT/CT approach allowed for the noninvasive serial analysis of peripheral angiogenesis in wild-type mice and mice that were endothelial nitric oxide synthase (eNOS) deficient (Fig. 1).

${ }^{99 m}$ Tc-NC100692 was also used successfully to quantitatively assess myocardial angiogenesis in mice lacking the gene for matrix metalloproteinase 9 (23) and in rats subjected to the local delivery of a human insulinlike growth factor 1 (IGF-1) gene by adeno-associated virus injected into the periinfarcted region (Fig. 2) (24). The availability of hybrid micro-SPECT/CT scanners and radiographic contrast agents with prolonged circulation times allowed delineation of the endocardial surface of the myocardium and improved the quantification of targeted molecular SPECT or PET images of the heart with CT reference images to define anatomic regions of interest. $\mathrm{Li}$ et al. developed a semiautomated approach for the absolute quantification of ${ }^{99 m} \mathrm{Tc}-\mathrm{NC} 100692$ uptake in the myocardium after myocardial infarction by using a hybrid microSPECT/CT approach $(25,26)$. Focal myocardial uptake estimated by in vivo hybrid imaging was correlated with uptake measured by $\gamma$-well counting. Targeted imaging of angiogenesis in patients has already been demonstrated to be feasible with SPECT of ${ }^{99 \mathrm{~m} T c-N C 100692}$ (Fig. 3) (27) and PET of an ${ }^{18}$ F-labeled RGD peptide (Fig. 4) (28).

Currently, there is great interest in the application of radiolabeled nanoparticles to the molecular imaging of angiogenesis (29). Through optimization of the nanoparticle composition and structure, it is possible to achieve 


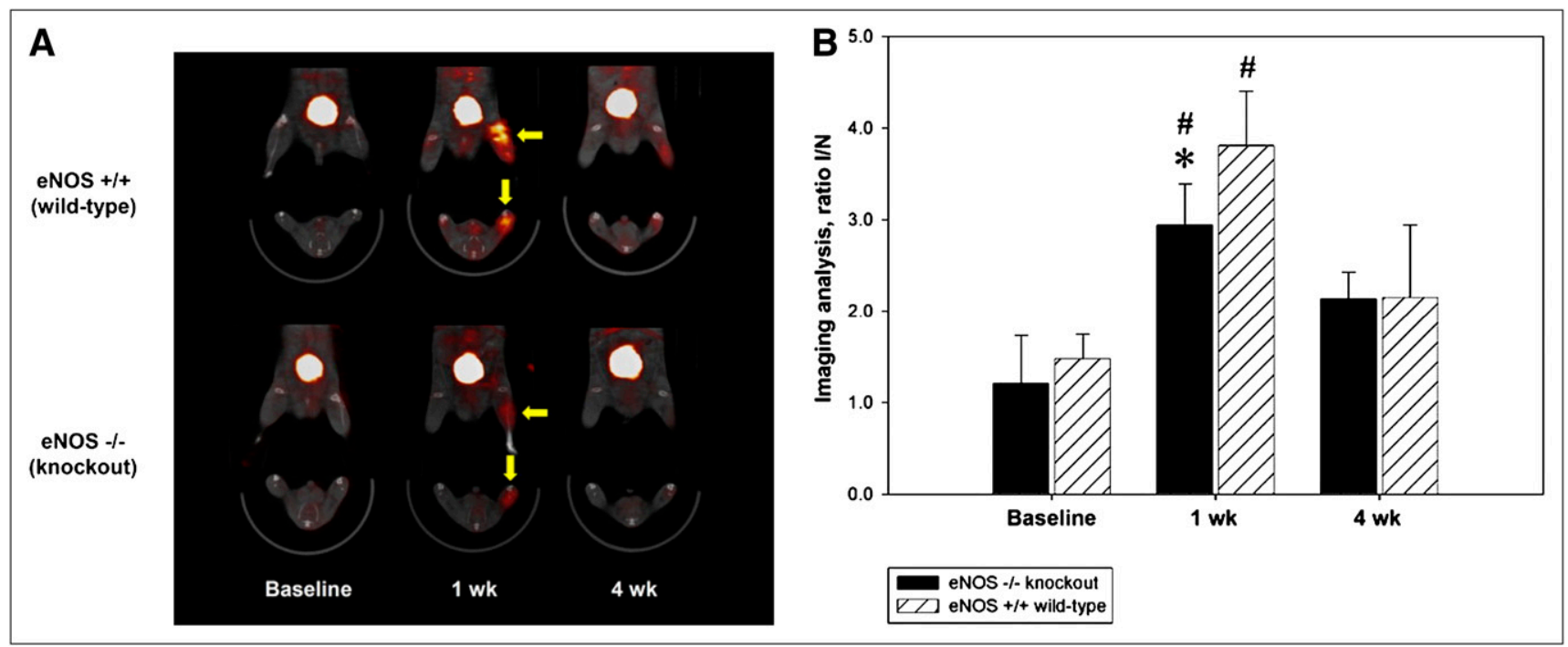

FIGURE 1. Analysis of wild-type $\left(\mathrm{eNOS}^{+/+}\right.$) and eNOS-deficient (eNOS ${ }^{-/-}$, or knockout) mice. (A) Representative microSPECT/CT images of eNOS ${ }^{+/+}$and eNOS ${ }^{-1-}$ mice injected with ${ }^{99 m T c-N C 100692 ~ a t ~ b a s e l i n e ~ a n d ~ a t ~} 7 \mathrm{~d}$ and 4 wk after right femoral artery ligation. Yellow arrows indicate ischemic regions with increased $99 \mathrm{mTC}-\mathrm{NC} 100692$ retention. Less retention was seen in eNOS ${ }^{-1-}$ mice. (B) Serial micro-SPECT/CT images were analyzed, and ischemic-to-nonischemic $99 \mathrm{mTc}$ TCN100692 activity ratios were calculated. Significant $(P<0.05)$ increase in ${ }^{99 m} \mathrm{Tc}-\mathrm{NC} 100692$ retention was seen in ischemic leg at $7 \mathrm{~d}$ after surgery in both groups. However, significantly less retention was seen at $7 \mathrm{~d}$ in eNOS ${ }^{-1-}$ mice than in wild-type mice. ${ }^{*} P<0.05$ for eNOS ${ }^{-1-}$ mice vs. wild-type mice; ${ }^{\#} P<0.05$ for value at $7 \mathrm{~d}$ vs. value at baseline. (Reprinted from (22).)

controlled in vivo circulation and tissue-specific targeting and render nanoparticles multimodal. The feasibility of noninvasive imaging of peripheral angiogenesis with nanoparticles labeled with a positron-emitting isotope was demonstrated in a murine model of hind-limb ischemia (30). The $\alpha_{\mathrm{v}} \beta_{3}$-integrin-targeted RGD nanoprobes consisted of a biodegradable dendritic core functionalized with a polyethylene oxide (PEO) chain that allowed for the modulation of pharmacokinetic properties. In that study, ${ }^{76} \mathrm{Br}$-labeled dendrimers were localized to angiogenic muscles within the ischemic murine hind limb (Fig. 5).

As outlined earlier, several radiolabeled ligands are being evaluated successfully in preclinical models of tumor and ischemia-induced peripheral and myocardial angiogenesis. These novel imaging approaches, in combination with techniques for quantifying the absolute uptake of targeted agents and assessing associated physiologic changes, may provide a new imaging strategy for evaluating genetic or stem cell therapies directed at stimulating angiogenesis in patients in clinical trials.

\section{ULTRASOUND APPROACHES}

Ultrasound is a relatively inexpensive technology, is currently the most widely used clinical cardiac imaging modality, and has a high temporal resolution, providing real-time imaging. In addition, ultrasound techniques have a high spatial resolution $(50-500 \mu \mathrm{m})$ but are subject to imaging artifacts and attenuation of ultrasound energy, which limits imaging in certain tissues, such as lung and bone. Molecular imaging with ultrasound relies on the application of targeted contrast agents. Contrast-enhanced ultrasound (CEU) is performed by the acoustic detection of microbubble or gas-containing nanoscale contrast agents. Ultrasound signals from these agents are produced by stable or inertial cavitation that occurs in the fluctuating pressures of the acoustic field. Sensitivity for CEU is difficult to define with conventional terms, such as molar concentration, but the technique is sufficiently sensitive to detect a single microbubble in tissue.

Ultrasound microbubble contrast agents are pure intravascular probes and uniquely target disease-related cellular and molecular processes within the vascular compartment only. Cellular targeting has been achieved either by changing the chemical constituents of the bubble shell to facilitate attachment to cells encountered in the presence of angiogenesis (i.e., leukocytes) or by conjugating specific ligands (i.e., antibodies, peptides, or peptidomimetic agents) to the surface of microbubbles $(31-33)$. These modifications cause microbubble binding to specific endothelial cell epitopes associated with the angiogenic process, resulting in a persistent contrast effect during ultrasound imaging $(34,35)$. For successful targeting of the vasculature, several factors should be considered, including the specificity and density of the target molecule, shear stress in the vasculature, and ligand density on microbubbles (31). Although the restriction to the intravascular compartment limits the scope of targets for the molecular imaging of angiogenesis, it also provides, in the case of endothelial cell targeting, confidence that signals from these agents are derived from 
A

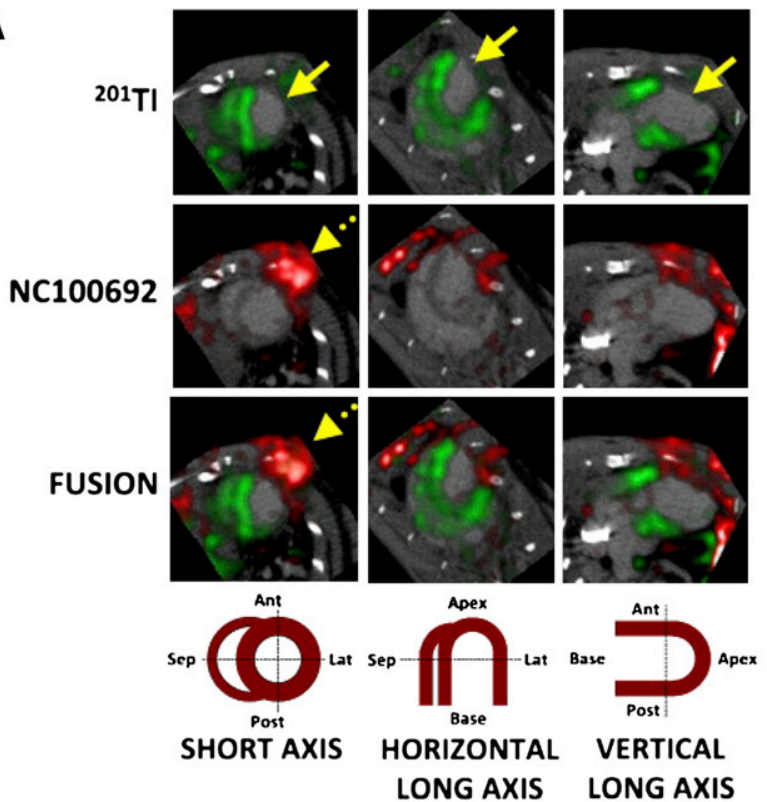

B
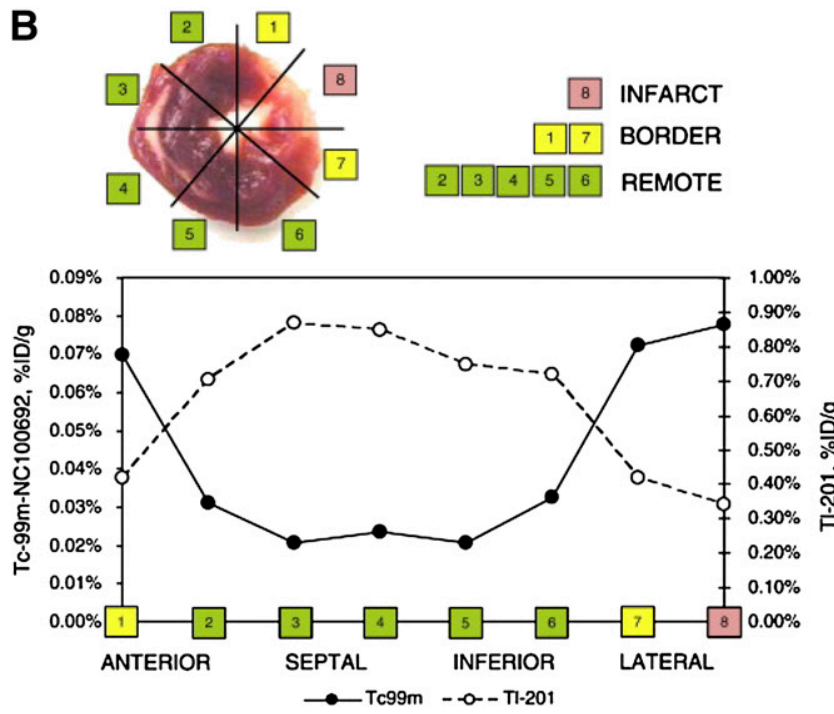

FIGURE 2. (A) In vivo micro-SPECT/CT images of ${ }^{201} \mathrm{TI}$ perfusion (top row, green) and 99mTC-NC100692 (middle row, red) in IGF-1 rat at 4 wk after myocardial infarction were reconstructed in short axis, horizontal long axis, and vertical long axis and fused (bottom row) with reference contrast CT image (gray scale). All rats had anterolateral ${ }^{201} \mathrm{Tl}$ perfusion defect (yellow solid arrows) and focal uptake of ${ }^{99 \mathrm{mT} T-}$ NC100692 in defect area after myocardial infarction. Contrast agent permitted better definition of myocardium and allowed differentiation of focal myocardial uptake of targeted radiotracer from uptake within chest wall at thoracotomy site (yellow dashed arrow). (B) Representative circumferential count profile of middle myocardial section of IGF-1 rat at 4 wk after myocardial infarction. Count profiles

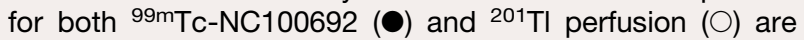
shown. $\% \mathrm{ID} / \mathrm{g}=$ percentage injected dose per gram. (Reprinted with permission of (24).)

endothelial cells rather than other cells active in vascular remodeling. Inherent in this technique are the facts that molecular imaging and quantitative perfusion imaging can be performed in a relatively rapid fashion and that the images can be spatially mapped to molecular signals.

Several studies have targeted $\alpha_{v} \beta_{3}$-integrin for the CEU imaging of angiogenesis. A microbubble contrast agent with conjugated RGD-bearing echistatin was initially used to evaluate $\alpha$-integrin expression in vivo in Matrigel (BD Biosciences) and in in situ glioma models of tumor angiogenesis (36,37). Using a similar approach, LeongPoi et al. were able to track peripheral angiogenesis in a rodent model of hind-limb ischemia (38). The angiogenesis was assessed noninvasively in control rats and animals treated with intramuscular sustained-release FGF 2 (38). Immediately after the iliac artery ligation and at subsequent intervals ranging from 4 to $28 \mathrm{~d}$, blood flow in the proximal adductor muscles was measured by $\mathrm{CEU}$ perfusion imaging. The targeted CEU imaging of $\alpha_{v} \beta_{3}$-integrin and $\alpha_{5} \beta_{1^{-}}$ integrin demonstrated that signals from targeted microbubbles were intense and peaked before changes in perfusion were seen, indicating that the heralding events in vascular remodeling could be detected by molecular imaging before major changes in perfusion were observed. FGF 2-treated muscle had a greater rate and extent of blood flow recovery

\section{A Short-axis}
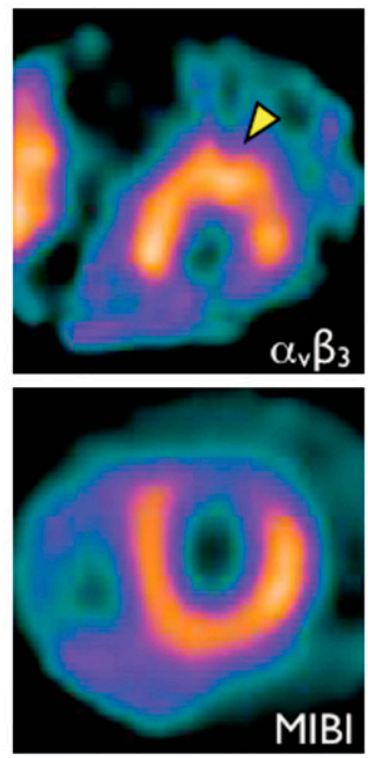

FIGURE 3. Noninvasive SPECT of angiogenesis in patient after percutaneous coronary intervention after myocardial infarction. After injection of $\alpha_{\mathrm{v}} \beta_{3}$-integrin-targeted agent (99mTc-NC100692) $3 \mathrm{wk}$ after myocardial infarction, focal signal enhancement (arrowheads) was noted in short-axis (A) and long-axis (B) views of infarct zone and confirmed by corresponding 99mTc-sestamibi (MIBI) perfusion images. (Reprinted with permission of (27). Images courtesy of Drs. Johan Verjans and Leonard Hofstra, University Hospital, Maastricht, The Netherlands.) 
FIGURE 4. Cardiac MRI with delayed enhancement (arrows) extending from anterior wall to apical region in 4-chamber (A) and 2-chamber (D) views. Identically reproduced location and geometry with severely reduced myocardial blood flow obtained with ${ }^{13} \mathrm{~N}$-ammonia, corresponding to regions of delayed enhancement on cardiac MRI (arrows) (B and E). Focal ${ }^{18} \mathrm{~F}-\mathrm{RGD}$ signal colocalized to infarcted area. This signal may reflect angiogenesis within healing area (arrows) (C and F). LA = left atrium; LV = left ventricle; $\mathrm{RA}=$ right atrium; $\mathrm{RV}=$ right ventricle. (Reprinted with permission of (28).)

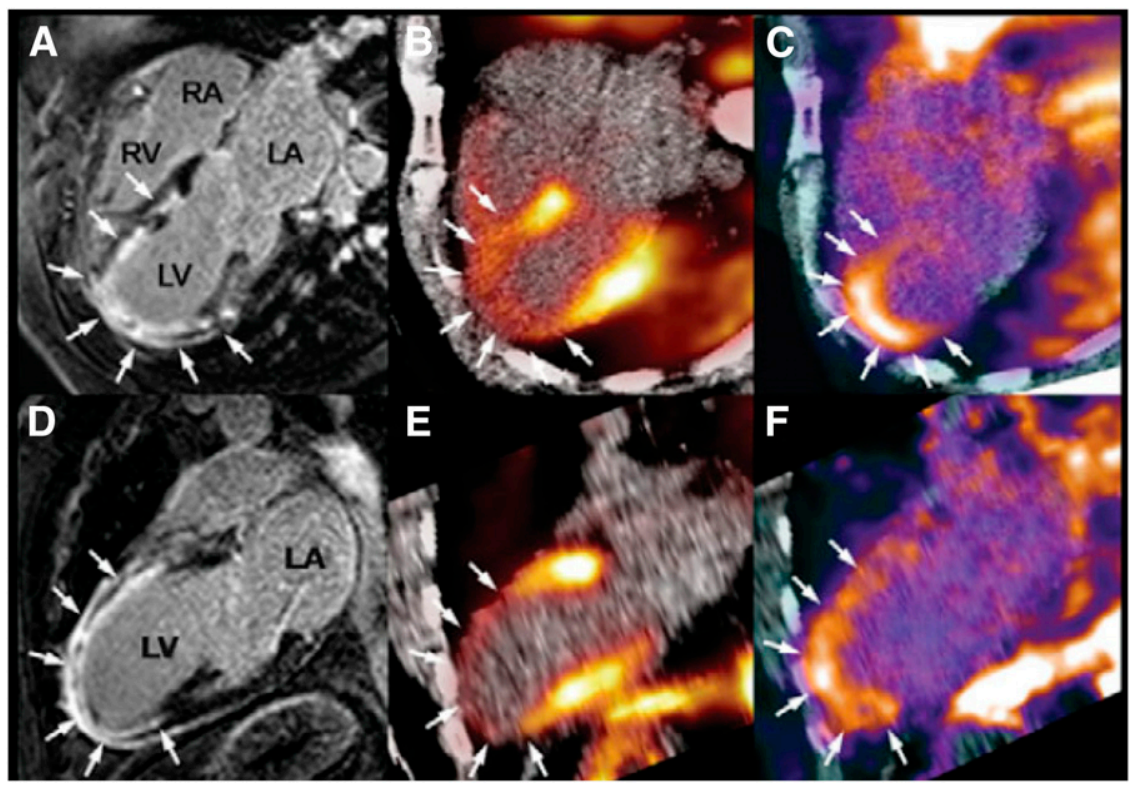

and a higher signal intensity from integrin-targeted microbubbles than nontreated muscle (Fig. 6).

Other groups have used different peptides and peptidomimetic agents conjugated to microbubbles to track the angiogenic process. Weller et al. demonstrated that a tumor endothelial cell binding-specific peptide sequence containing an RRL (arginine-arginine-leucine) motif can be successfully used to image tumor angiogenesis (39). Although the mechanism of attachment of the microbubbles to the vascular wall for this molecule is unclear, most likely it involves direct attachment to the endothelial cell surface
(33). More recently, microbubbles targeted to growth factor receptors expressed during vascular remodeling were tested for the imaging of tumor and peripheral angiogenesis (Fig. 7). Targeted microbubbles were developed specifically to bind to VEGF receptor 2 (VEGFR2) (by use of antiVEGFR2 antibodies and the biotin-streptavidin interaction), which is upregulated on endothelial cells of tumor blood vessels (40). VEGFR2-targeted microbubbles, control microbubbles, and nonlabeled microbubbles were tested for binding specificity on cells expressing VEGFR2 (mouse angiosarcoma SVR cells) and control cells. In vivo
A

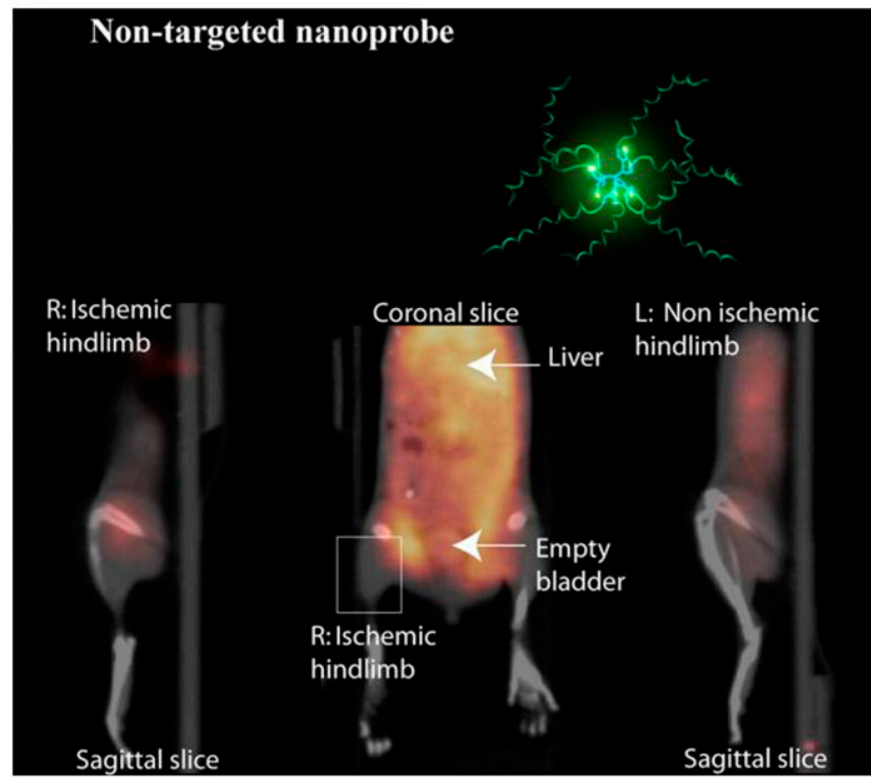

B

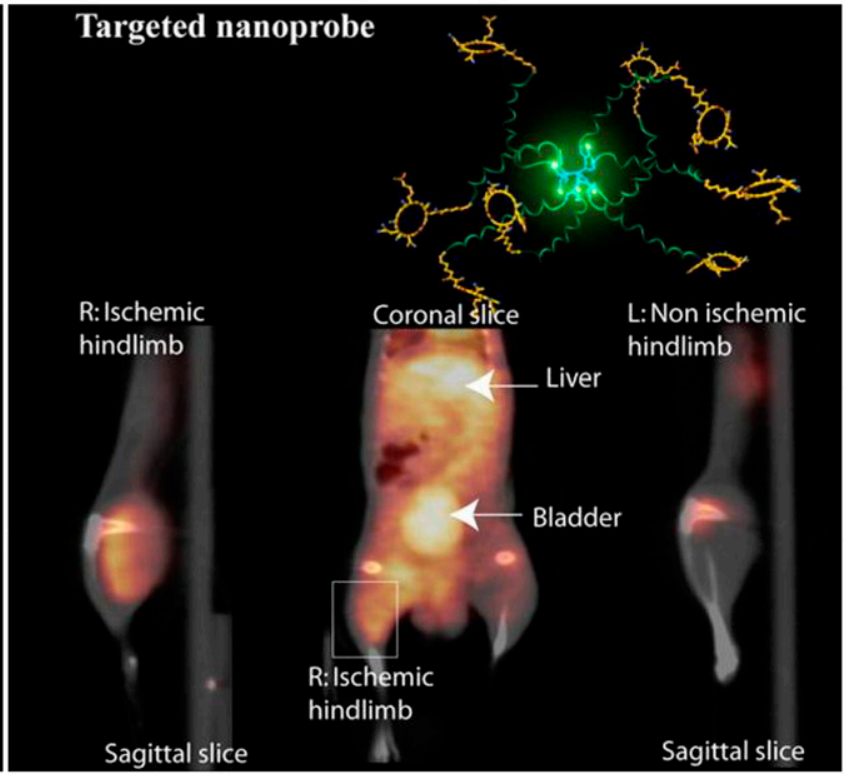

FIGURE 5. Noninvasive PET/CT images of angiogenesis induced by hind-limb ischemia in murine model. (A) Nontargeted dendritic nanoprobes (bottom center). (B) Uptake of $\alpha_{v} \beta_{3}$-targeted dendritic nanoprobes was higher in ischemic hind limb (left side of image) than in control hind limb (right side of image). (Reprinted with permission of (30).) 


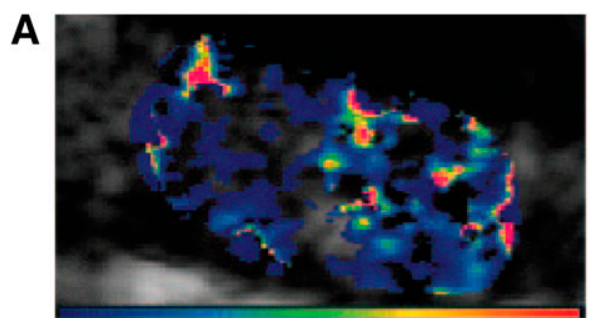

Control

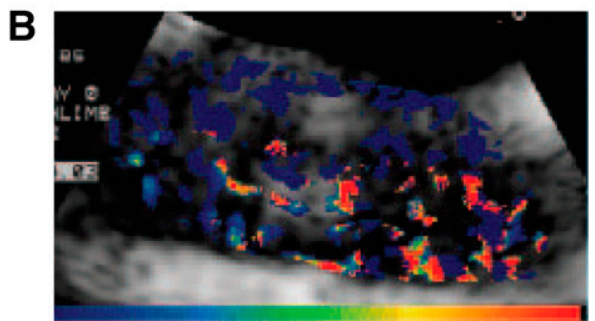

Control

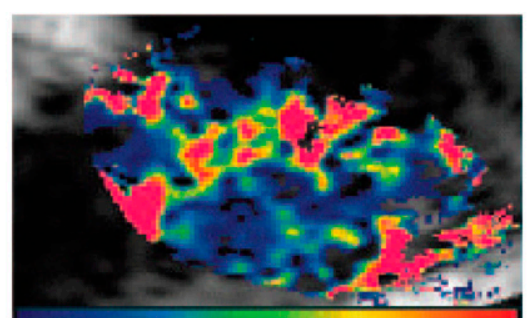

Ischemic

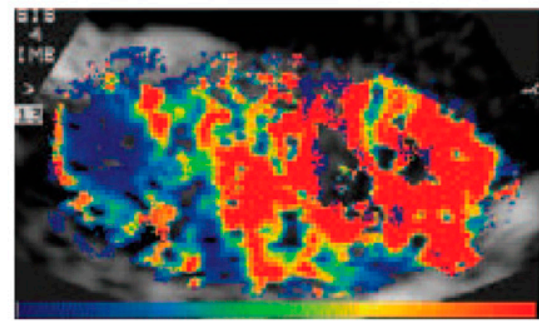

Ischemic
FIGURE 6. Examples of color-coded CEU images reflecting retention fraction of integrin-targeted microbubbles in control and ischemic proximal hind-limb adductor muscles from untreated $(A)$ and FGF 2-treated (B) rat $4 \mathrm{~d}$ after iliac artery ligation. Color scales are shown at bottom. (Reprinted with permission of (38).)
CEU imaging showed significantly higher average image intensity with targeted microbubbles than with control microbubbles for the evaluation of angiosarcoma and malignant glioma tumors.

Although inflammation is often implicated by the formation of neovessels, targeted imaging of leukocyte adhesion molecules can also be used to identify angiogenesis $(32,34,41)$. Preliminary studies in a rat model of middle cerebral artery occlusion demonstrated that microbubbles with surfaces modified by phosphatidylserine can be used to detect acute inflammatory responses associated with acute ischemia-reperfusion injury of the brain (33). More recently, Behm et al. demonstrated that ultrasound microbubbles with surfaces modified to target activated neutrophils, $\alpha_{5}$-integrin, and VCAM-1 can successfully image different components of inflammatory responses that par- ticipate in vascular development and remodeling (42). These studies established that the physiologic link between the immune response and angiogenesis, which is a target for emerging therapies for ischemic disease, can be assessed with molecular and cellular imaging.

The feasibility of targeting ultrasound contrast agents to disease-related markers, including angiogenesis, has been firmly established, and this technique is currently being applied as a high-throughput research tool to define pathophysiologic processes in both the spatial and the temporal domains. Although initial steps are currently being made toward the development of targeted agents suitable for clinical applications, the potential clinical role of this technology is contingent on further refinement of targeted microbubble chemistry. Moreover, there is a need for studies indicating that the unique diagnostic information
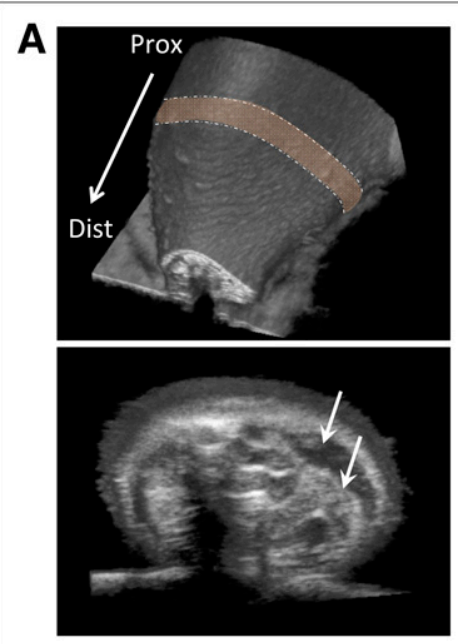
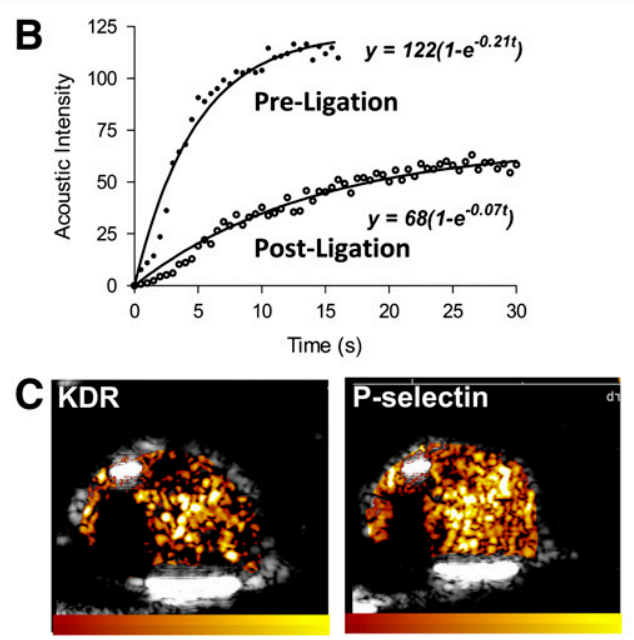

FIGURE 7. Contrast ultrasound imaging of angiogenic endothelial cell phenotype in chronic limb ischemia. (A) High-frequency (30-MHz) 3D ultrasound rendering of mouse hind limb with single short-axis elevational plane (bottom) showing region of proximal (Prox) adductor muscle group (arrows). Dist $=$ distal. (B) Perfusion imaging data from adductor muscle group before and after iliac ligation in which product of rate constant and plateau intensity of curves denotes blood flow and is reduced by $80 \%$ during acute ischemia but improves to $20 \%$ of normal by $21 \mathrm{~d}$. (C) Molecular imaging with microbubbles targeted to kinase insert domain receptor (VEGFR-2) and to P-selectin at day 3 after production of ischemia. 
obtained from molecular CEU imaging can positively affect patient care.

\section{MAGNETIC RESONANCE APPROACHES}

Contrary to the acquisition of electric signals from the body, the recording of magnetic signals is less easily accomplished because of the low strength of these signals, requiring strong magnets, manipulation of the signal source, and extremely sensitive sensors. However, MRI provides high spatial and temporal resolution as well as excellent soft-tissue contrast without the use of ionizing radiation or contrast agents. Moreover, anatomic information can be coregistered with functional and molecular information within a single imaging modality. Unfortunately, MRI has relatively low sensitivity for the detection of targeted agents, but this limitation might be overcome by signal amplification techniques that provide higher signalto-background ratios (43).

The ${ }^{1} \mathrm{H}$ proton is the most commonly imaged nucleus because of its high gyromagnetic ratio and its high concentration in biologic tissues. For targeted imaging applications, however, another naturally occurring nucleus, ${ }^{19} \mathrm{~F}$, has several attractive characteristics. The gyromagnetic ratio of ${ }^{19} \mathrm{~F}$ is close to that of ${ }^{1} \mathrm{H}$, but in biologic tissues, the concentration of ${ }^{19} \mathrm{~F}$ is low, ensuring low background levels for targeted imaging with particles that contain ${ }^{19} \mathrm{~F}$. Thus, ${ }^{19} \mathrm{~F}$ spectroscopy of targeted perfluorocarbon particles is an attractive permutation of molecular MRI that offers high signal-to-noise ratios. Typically, the particles consist of perfluorocarbon emulsions encapsulated in a lipid shell with excellent biocompatibility (44). No toxicity, carcinogenicity, mutagenicity, or teratogenic effects have been reported for pure fluorocarbons that have molecular weights of 460-520 and that are biologically inert, removed through the reticuloendothelial system, and excreted primarily through the lungs and in small amounts through the skin $(44,45)$. Indeed, CEU makes use of clinically approved microparticles that often contain perfluoro gas in a stabilizing shell (denatured albumin, surfactants, or phospholipids) (46), further attesting to the biocompatibility of perfluorocarbons.

In contrast-enhanced MRI studies, the strong magnetization of magnetic particles is used to enhance the signals resulting from the intrinsic magnetization of the nuclei in the body. Naturally occurring contrast can be modified by proximity to exogenous materials. Commonly used examples are compounds containing gadolinium $(\mathrm{Gd})$ or iron oxides. Gd is a paramagnetic agent that brightens regions retaining the contrast agent in T1-weighted images. Iron oxides are superparamagnetic agents that change magnetic susceptibility, producing local field gradients that increase the rate of dephasing and thus darken affected voxels in $\mathrm{T} 2 *$-sensitive images.

Historically, the first effort at targeted imaging of angiogenesis was achieved with $\mathrm{Gd}^{3+}$-containing para- magnetic liposomes (300 $\mathrm{nm}$ in diameter) conjugated to an $\alpha_{\mathrm{v}} \beta_{3}$-integrin antibody (LM609) as a ligand (12). This approach allowed for the imaging of rabbit carcinomas through direct targeting of the paramagnetic agent to the angiogenic vasculature. Another group used magnetic nanoparticles conjugated to a peptidomimetic $\alpha_{\mathrm{v}} \beta_{3}$-integrin antagonist for the imaging of squamous cell carcinomas with a clinical 1.5-T MRI scanner (47). The T1-weighted signal was significantly increased in the periphery of the tumor as early as $2 \mathrm{~h}$ after injection. Moreover, melanoma tumor xenografts were also successfully imaged in mice with $\alpha_{v} \beta_{3}$-integrin-targeted paramagnetic nanoparticles, further attesting to the characterization of early melanomas with MRI. More recently, Kluza et al. investigated the targeting efficacy of liposomal nanoparticles that were functionalized with 2 angiogenesis-specific targeting ligands, $\alpha_{v} \beta_{3}$-integrin-specific RGD and galectin 1-specific peptides (48). They used bimodal (MRI- and fluorescencedetectable) liposomal nanoparticles as a contrast agent, which allowed for both high-sensitivity (fluorescence) and high-resolution (MRI) assessments of the angiogenic process. Moreover, this multitargeting approach produced a synergistic targeting effect causing a level of retention of nanoparticles that was significantly higher than that obtained with single-ligand targeting (48).

The use of Gd-based contrast agents in molecular targeted MRI is limited by relatively low sensitivity in the millimolar range. Therefore, novel techniques to increase signal-to-background contrast ratios were recently introduced. Superparamagnetic iron oxide (SPIO) nanoparticles provide much higher sensitivity because iron oxide leads to decreases in signals in $\mathrm{T} 2$-weighted and especially $\mathrm{T} 2 *$-weighted sequences, resulting in "negativecontrast" imaging (49). Zhang et al. successfully used ultrasmall RGD-conjugated SPIO nanoparticles targeted to $\alpha_{v} \beta_{3}$-integrin for the $\mathrm{T} 2 *$-weighted imaging of tumor vasculature with a clinical 1.5-T MRI scanner (50). Shortly after injection of the RGD-SPIO nanoparticles, a significant irregular decrease in the signal density was observed within $\alpha_{v} \beta_{3}$-integrin-positive tumor vessels compared with control tumor vessels. To take advantage of the strengths of MRI (high resolution) and functional modalities (high sensitivity with nuclear or optical techniques), some groups have focused on the design of multimodal iron oxide-based nanoparticles. Montet et al. proposed using RGD-cross-linked iron oxide-Cy5.5 nanoparticles for both high-resolution MRI and high-sensitivity fluorescence imaging of angiogenesis (51). A similar approach was proposed by Dijkgraaf et al. for tumor detection with a high degree of accuracy (20). Both small-animal PET and T2weighted MRI showed integrin-specific delivery of polyaspartic acid-coated iron oxide nanoparticles coupled to RGD and DOTA as a linker to a positron-emitting radioisotope (20).

MR images have been quantified by reporting a difference in signal intensities between baseline images and 


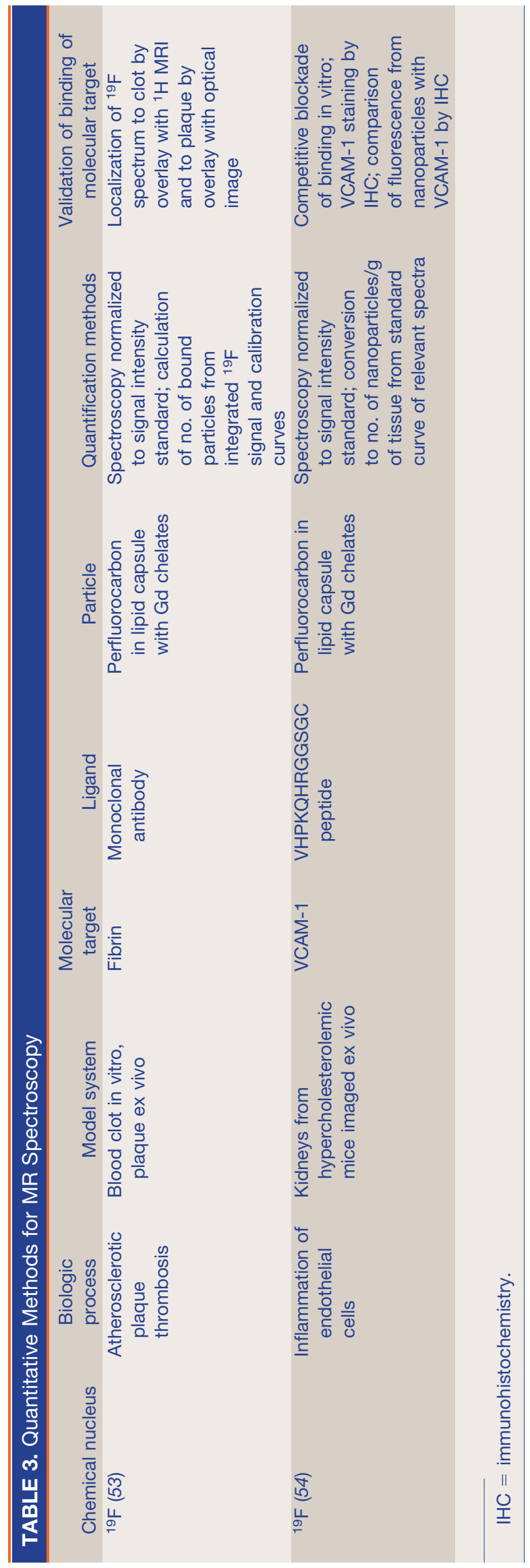

images acquired after the injection of molecular imaging probes. Table 3 and Table 4 summarize studies in which targeted molecular imaging has been combined with quantitative methods to determine the extent of nanoparticle binding to the molecular target. Most examples are pertinent to molecular imaging in the cardiovascular system and used ${ }^{19} \mathrm{~F}$ spectroscopy or $\mathrm{T} 1$-weighted imaging of Gd chelates, but an example pertaining to lipoprotein metabolism was added because it provides an elegant example of molecular imaging with iron oxide nanoparticles and quantitative methods (52).

In a study aimed at detecting fibrin as a marker of thrombosis in atherosclerotic plaque, Morawski et al. used perfluorocarbon nanoparticles targeted to fibrin with a monoclonal antibody (53). The lipid monolayer encapsulating the perfluorocarbon emulsion contained $\mathrm{Gd}$ chelates, and the amount of $\mathrm{Gd}$ was used to validate the ${ }^{19} \mathrm{~F}$ signals and to confirm that the particles had bound to fibrin. The ${ }^{19} \mathrm{~F}$ signals from the experimental samples were integrated with respect to the trichlorofluoromethane measurements, and the integrated values were plotted against the amount of perfluorocarbon in each sample and fit with linear regression. The amount of nanoparticles bound to fibrin was derived from ${ }^{19} \mathrm{~F}$ spectroscopy, and this measurement was further validated by measuring the amount of $\mathrm{Gd}$ in each sample by use of neutron activation techniques. In another experiment, members of the same group quantified the binding of perfluorocarbon nanoparticles to VCAM-1 in the kidneys of hypercholesterolemic mice (54). The nanoparticles contained crown ether (CE) as a source of ${ }^{19} \mathrm{~F}$, and for ${ }^{19} \mathrm{~F}$ signal calibration and quantification, a reference sample of $10 \mu \mathrm{L}$ of $1 \%$ perfluorooctylbromide (PFOB) in a capillary tube was included with each measurement. The PFOB signal was clearly distinct from the $\mathrm{CE}$ signal, allowing for the calibration of the $\mathrm{CE}$ signal against a known quantity of PFOB. Absolute nanoparticle numbers per tissue wet weight were calculated from a standard curve of relative signal peak areas corresponding to a range of $\mathrm{CE}$ and $\mathrm{PFOB}$ emulsion concentrations.

Two examples of T1-weighted imaging of targeted nanoparticles containing $\mathrm{Gd}$ chelates are also shown in Table 4. In an experiment aimed at the imaging of angiogenesis in a rabbit model of atherosclerosis, Winter et al. (55) and Wickline et al. (56) used perfluorocarbon nanoparticles with Gd chelates in the lipid shell of the particles (Fig. 8). The particles were targeted to $\alpha_{\mathrm{v}} \beta_{3}$-integrin by a peptidomimetic agent with the RGD peptide sequence, and T1-weighted relaxivity was measured before and after injection of the targeted nanoparticles. The animals were imaged together with a reference sample that contained a known amount of Gd chelate and that was also placed in the field of view.

Oostendorp et al. used paramagnetic quantum dots to target CD13/aminopeptidase $\mathrm{N}$, a marker of angiogenic endothelium in a murine tumor model, and correlated the 


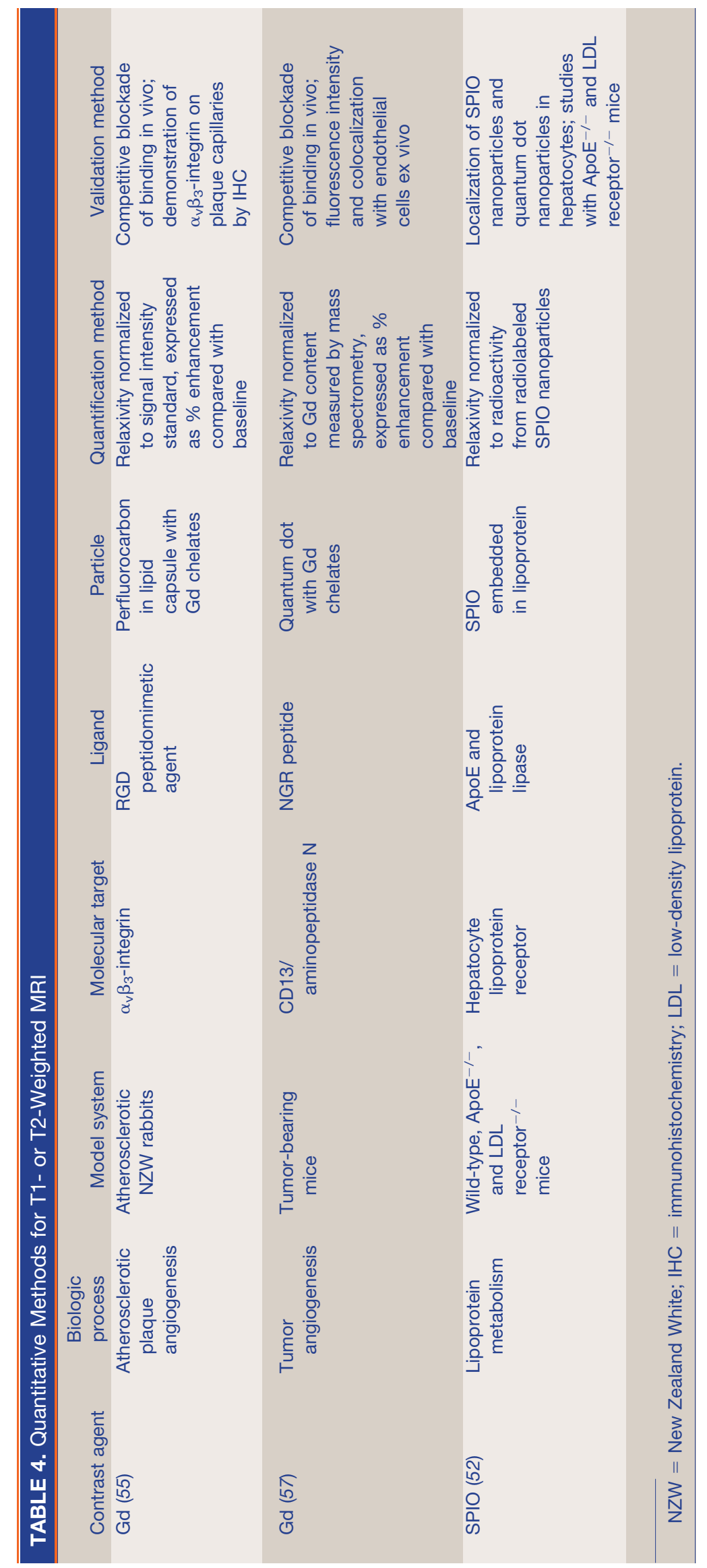


results with absolute Gd content (57). Finally, using T2*weighted imaging, Bruns et al. tracked lipoprotein metabolism with targeted nanoparticles containing SPIO (52). The particles were targeted to the hepatocyte lipoprotein receptor, and the ligands were apolipoprotein E (ApoE) and lipoprotein lipase. That study is of further interest because the authors demonstrated that there was a quantifiable difference in signals between mice that had ApoE and lipoprotein lipase on hepatocytes and animals that lacked either ApoE or the low-density lipoprotein receptor, demonstrating the ability of molecular MRI to quantitatively discriminate between biologic conditions with different hepatic lipoprotein uptake kinetics.

Despite progress in the quantification of MR signals emanating from targeted molecular probes, there are still many sources of potential error; thus far, most molecular MRI studies have been qualitative or, like those summarized here, semiquantitative (58). Quantitative accuracy and reproducibility need to be further established before molecular MRI methods can be standardized and crossvalidated. Even when signal changes have been calibrated to standard curves, many sources of error remain. For example, variations in tissue $\mathrm{O}_{2}$ concentrations can influence signals from perfluorocarbons (54). As shown by the studies summarized here, determination of the absolute contrast agent concentration requires calibration and is susceptible to flip-angle errors (58). For T1 measurements, Schabel and Parker showed that one of the most commonly used pulse sequences, that is, the spoiled gradient-echo sequence, is highly angle dependent; they demonstrated the existence of a concentration- and tissue-dependent optimal flip angle that minimizes concentration uncertainty and that is significantly larger than the corresponding Ernst angle (59).

Iron oxides are attractive for molecular MRI applications because of their favorable toxicity profile compared with that of $\mathrm{Gd}$ chelates, but estimation of the hypointensity indicated by cells targeted or labeled with SPIO is highly dependent on imaging parameters, pulse sequences, field strengths, and voxel volumes. For example, when imaging cells labeled with SPIO, Liu et al. showed that T2* values from shifted spin-echo ultrashort $\mathrm{T} 2 *$ mapping were approximately $10 \%$ higher than values from ultrashortecho-time imaging (60). The concentration of particles inside a cell, in itself, strongly influences relaxivity. Bowen et al. found that the compartmentalization of SPIO inside cells significantly increased relative $\mathrm{T} 2 *$ sensitivity compared with uniformly distributed iron oxide (61). Thus, whether a targeted molecular imaging SPIO probe is internalized or not will strongly affect the relaxivity measurements obtained from such a probe. The density of the probe on the cell surface is also bound to influence the relaxivity changes induced by such a probe.

Other factors that influence signal quantification from molecular MRI probes are blood flow and pressure within target tissues; for example, vascularized tumors are char-

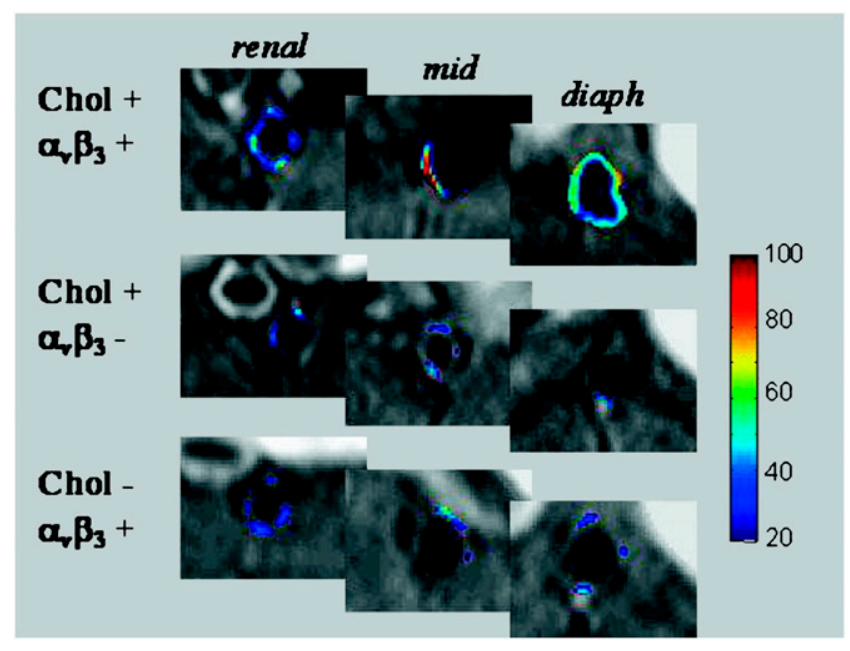

FIGURE 8. Angiogenesis induced in cholesterol (Chol)-fed rabbits. Aortic cross sections were imaged at $1.5 \mathrm{~T}$ with $\alpha_{v} \beta_{3}$-integrin-targeted nanoparticles. Note heterogeneous distribution in aortic cross sections (false colored contrast enhancement) but little enhancement in nontargeted rabbits $\left(\alpha_{v} \beta_{3}-\right)$ or rabbits on standard diet $(\mathrm{Chol}-)$. diaph $=$ diaphragm; mid = mid-aorta. (Reprinted with permission of (56).)

acterized by areas of stasis, which will favor the aggregation of nanoparticles, which may, in turn, induce relaxivity changes similar to those mentioned for intracellular compartmentalization. Indeed, flow in tumors is highly heterogeneous and likely to further confound quantitative assessments of nanoparticle binding to tumor vessels (62).

\section{CONCLUSION}

The future for the noninvasive imaging of angiogenesis may rest on the development of improved targeted biologic markers of angiogenesis and multimodality imaging. VEGF receptors could serve as targets for the imaging of angiogenesis by imparting physiologic information on hypoxic stress. $\alpha_{\mathrm{v}} \beta_{3}$-integrin appears to be another important target for the imaging of angiogenesis. Several radiolabeled ligands targeted to $\alpha_{\mathrm{v}} \beta_{3}$-integrin have already proved useful for tracking angiogenesis in experimental models of myocardial ischemia and hind-limb ischemia. The relatively high sensitivity of radiotracer-based imaging systems, such as SPECT and PET, has provided an advantage in the practical application of molecular imaging techniques for the evaluation of angiogenesis; such systems have already progressed to clinical trials. These techniques will need to be combined with higher-resolution imaging methods, such as CT and MRI, to facilitate accurate imaging quantification.

Targeted ultrasound imaging of angiogenesis is feasible and offers the potential for high-throughput screening of molecular and pathophysiologic processes. Although initial steps toward the development of targeted agents suitable for clinical applications are currently being made, the potential 
clinical role of this technology is contingent on further refinement of targeted microbubble chemistry. Targeted MRI of angiogenesis offers potential advantages regarding resolution and integration with metabolic or physiologic indices, such as perfusion and permeability; however, current methods lack sensitivity and will require clinical validation.

All of the clinical imaging modalities have advantages and disadvantages for the targeted molecular imaging of angiogenesis. Therefore, the selection of an imaging modality should be based on the accessibility of instrumentation, the availability of targeted probes, and the properties of the biologic system to be studied. The optimal application of any targeted imaging approach will require registration with physiologic and anatomic images and image quantification. The application of nanotechnology to angiogenesis imaging is an exciting new area of research, but further studies are needed to assess the feasibility of applying this technology to the clinical arena. The targeted imaging approaches outlined in this review offer the potential to evaluate new pharmacologic or cell-based therapies directed at stimulating angiogenesis and arteriogenesis and may lead to more individualized care of patients with ischemic heart disease.

\section{ACKNOWLEDGMENTS}

This work was supported in part by grants JDRF 102006-726, R01 HL078622, and R01 HL065662.

\section{REFERENCES}

1. Fam NP, Verma S, Kutryk M, Stewart DJ. Clinician guide to angiogenesis. Circulation. 2003;108:2613-2618.

2. Sinusas A. Imaging of angiogenesis. J Nucl Cardiol. 2004;11:617-633.

3. Simons M, Bonow R, Chronos N, et al. Clinical trials in coronary angiogenesis: issues, problems, consensus-an expert panel summary. Circulation. 2000;102: E73-E86.

4. Lake Tahoe invitation meeting 2002. J Nucl Cardiol. 2003;10:223-257.

5. Collingridge D, Carroll V, Glaser M, et al. The development of $\left[{ }^{124} \mathrm{I}\right]$ iodinatedVG76e: a novel tracer for imaging vascular endothelial growth factor in vivo using positron emission tomography. Cancer Res. 2002;62:5912-5919.

6. Li S, Peck-Radosavljevic M, Koller E, et al. Characterization of ${ }^{123}$ I-vascular endothelial growth factor-binding sites expressed on human tumour cells: possible implication for tumour scintigraphy. Int J Cancer. 2001;91:789-796.

7. Cai W, Chen K, Mohamedali K, et al. PET of vascular endothelial growth factor receptor expression. J Nucl Med. 2006;47:2048-2056.

8. Rodriguez-Porcel M, Cai W, Gheysens O, et al. Imaging of VEGF receptor in a rat myocardial infarction model using PET. J Nucl Med. 2008;49:667-673.

9. Backer M, Levashova Z, Patel V, et al. Molecular imaging of VEGF receptors in angiogenic vasculature with single-chain VEGF-based probes. Nat Med. 2007; 13:504-509.

10. Levashova Z, Backer M, Backer J, Blankenberg F. Imaging vascular endothelial growth factor (VEGF) receptors in turpentine-induced sterile thigh abscesses with radiolabeled single-chain VEGF. J Nucl Med. 2009;50:2058-2063.

11. Wagner B, Anton M, Nekolla S, et al. Noninvasive characterization of myocardial molecular interventions by integrated positron emission tomography and computed tomography. J Am Coll Cardiol. 2006;48:2107-2115.

12. Sipkins D, Cheresh D, Kazemi M, Nevin L, Bednarski M, Li K. Detection of tumor angiogenesis in vivo by alphaVbeta3-targeted magnetic resonance imaging. Nat Med. 1998;4:623-626.

13. Haubner R. Noninvasive tracer techniques to characterize angiogenesis. Handb Exp Pharmacol. 2008(185 Pt 2):323-339.
14. Harris T, Kalogeropoulos S, Nguyen T, et al. Design, synthesis, and evaluation of radiolabeled integrin alpha $\mathrm{v}$ beta 3 receptor antagonists for tumor imaging and radiotherapy. Cancer Biother Radiopharm. 2003;18:627-641.

15. Sadeghi MM. Imaging $\alpha v \beta 3$ integrin in vascular injury: does this reflect increased integrin expression or activation? [abstract]. Circulation. 2003;108(17 suppl): 1868 .

16. Meoli DF, Sadeghi MM, Krassilnikova S, et al. Noninvasive imaging of myocardial angiogenesis following experimental myocardial infarction. J Clin Invest. 2004;113:1684-1691.

17. Kalinowski L, Dobrucki L, Meoli D, et al. Targeted imaging of hypoxia-induced integrin activation in myocardium early after infarction. J Appl Physiol. 2008;104:1504-1512.

18. Liu S, Liu Z, Chen K, et al. ${ }^{18}$ F-labeled galacto and PEGylated RGD dimers for PET imaging of alpha(v)beta(3) integrin expression. Mol Imaging Biol. 2009 [Epub ahead of print].

19. Liu S. Radiolabeled cyclic RGD peptides as integrin alpha(v)beta(3)-targeted radiotracers: maximizing binding affinity via bivalency. Bioconjug Chem. 2009; 20:2199-2213.

20. Dijkgraaf I, Beer A, Wester H. Application of RGD-containing peptides as imaging probes for alphavbeta3 expression. Front Biosci. 2009;14:887-899.

21. Hua J, Dobrucki LW, Sadeghi MM, et al. Noninvasive imaging of angiogenesis with a $99 \mathrm{~m}$ Tc-labeled peptide targeted at alphavbeta3 integrin after murine hindlimb ischemia. Circulation. 2005;111:3255-3260.

22. Dobrucki L, Dione D, Kalinowski L, et al. Serial noninvasive targeted imaging of peripheral angiogenesis: validation and application of a semiautomated quantitative approach. J Nucl Med. 2009;50:1356-1363.

23. Lindsey M, Escobar G, Dobrucki L, et al. Matrix metalloproteinase-9 gene deletion facilitates angiogenesis after myocardial infarction. Am J Physiol Heart Circ Physiol. 2006;290:H232-H239.

24. Dobrucki LW, Tsutsumi Y, Kalinowski L, et al. Analysis of angiogenesis induced by local IGF-1 expression after myocardial infarction using microSPECT-CT imaging. J Mol Cell Cardiol. October 20, 2009 [Epub ahead of print].

25. Li S, Dobrucki LW, Sinusas AJ, Liu YH. A new method for SPECT quantification of targeted radiotracers uptake in the myocardium. Med Image Comput Comput Assist Interv. 2005;8:684-691.

26. Li S, Dobrucki WL, Aikawa R, Mendizabal M, Sinusas AJ, Liu Y. A new method for quantification of targeted radiotracer uptake from cardiac microSPECT/CT images: a rat validation. J Nucl Med. 47(suppl 1):253P.

27. Jaffer F, Libby P, Weissleder R. Molecular imaging of cardiovascular disease. Circulation. 2007;116:1052-1061.

28. Makowski M, Ebersberger U, Nekolla S, Schwaiger M. In vivo molecular imaging of angiogenesis, targeting alphavbeta3 integrin expression, in a patient after acute myocardial infarction. Eur Heart J. 2008;29:2201.

29. Welch M, Hawker C, Wooley K. The advantages of nanoparticles for PET. $J$ Nucl Med. 2009;50:1743-1746.

30. Almutairi A, Rossin R, Shokeen M, et al. Biodegradable dendritic positronemitting nanoprobes for the noninvasive imaging of angiogenesis. Proc Natl Acad Sci USA. 2009;106:685-690.

31. Kaufmann B, Lindner J. Molecular imaging with targeted contrast ultrasound. Curr Opin Biotechnol. 2007;18:11-16.

32. Lindner J. Contrast ultrasound molecular imaging of inflammation in cardiovascular disease. Cardiovasc Res. 2009;84:182-189.

33. Piedra M, Allroggen A, Lindner J. Molecular imaging with targeted contrast ultrasound. Cerebrovasc Dis. 2009;27(suppl 2):66-74.

34. Sinusas A, Bengel F, Nahrendorf $M$, et al. Multimodality cardiovascular molecular imaging, part I. Circ Cardiovasc Imaging. 2008;1:244-256.

35. Saraste A, Nekolla S, Schwaiger M. Cardiovascular molecular imaging: an overview. Cardiovasc Res. 2009;83:643-652.

36. Ellegala D, Leong-Poi H, Carpenter J, et al. Imaging tumor angiogenesis with contrast ultrasound and microbubbles targeted to alpha(v)beta3. Circulation. 2003; 108:336-341.

37. Leong-Poi H, Christiansen J, Klibanov A, Kaul S, Lindner J. Noninvasive assessment of angiogenesis by ultrasound and microbubbles targeted to alpha(v)integrins. Circulation. 2003;107:455-460.

38. Leong-Poi H, Christiansen J, Heppner P, et al. Assessment of endogenous and therapeutic arteriogenesis by contrast ultrasound molecular imaging of integrin expression. Circulation. 2005;111:3248-3254.

39. Weller G, Wong M, Modzelewski R, et al. Ultrasonic imaging of tumor angiogenesis using contrast microbubbles targeted via the tumor-binding peptide arginine-arginine-leucine. Cancer Res. 2005;65:533-539.

40. Willmann J, Paulmurugan R, Chen K, et al. US imaging of tumor angiogenesis with microbubbles targeted to vascular endothelial growth factor receptor type 2 in mice. Radiology. 2008;246:508-518. 
41. Nahrendorf M, Sosnovik D, French B, et al. Multimodality cardiovascular molecular imaging, part II. Circ Cardiovasc Imaging. 2009;2:56-70.

42. Behm C, Kaufmann B, Carr C, et al. Molecular imaging of endothelial vascular cell adhesion molecule-1 expression and inflammatory cell recruitment during vasculogenesis and ischemia-mediated arteriogenesis. Circulation. 2008;117:2902-2911.

43. Jaffer F, Weissleder R. Seeing within: molecular imaging of the cardiovascular system. Circ Res. 2004;94:433-445.

44. Tran T, Caruthers S, Hughes M, et al. Clinical applications of perfluorocarbon nanoparticles for molecular imaging and targeted therapeutics. Int J Nanomedicine. 2007;2:515-526.

45. Joseph P, Yuasa Y, Kundel H, Mukherji B, Sloviter H. Magnetic resonance imaging of fluorine in rats infused with artificial blood. Invest Radiol. 1985;20:504-509.

46. Cosgrove D. Ultrasound contrast agents: an overview. Eur J Radiol. 2006; 60:324-330.

47. Winter PM, Caruthers SD, Kassner A, et al. Molecular imaging of angiogenesis in nascent $\mathrm{Vx}$-2 rabbit tumors using a novel alpha(nu)beta3-targeted nanoparticle and 1.5 tesla magnetic resonance imaging. Cancer Res. 2003;63:5838-5843.

48. Kluza E, van der Schaft DW, Hautvast PA, et al. Synergistic targeting of alpha(v)beta(3) integrin and galectin-1 with heteromultivalent paramagnetic liposomes for combined MR imaging and treatment of angiogenesis. Nano Lett. 2010;10:52-58.

49. Thorek D, Chen A, Czupryna J, Tsourkas A. Superparamagnetic iron oxide nanoparticle probes for molecular imaging. Ann Biomed Eng. 2006;34:23-38.

50. Zhang C, Jugold M, Woenne E, et al. Specific targeting of tumor angiogenesis by RGD-conjugated ultrasmall superparamagnetic iron oxide particles using a clinical 1.5-T magnetic resonance scanner. Cancer Res. 2007;67:1555-1562.

51. Montet X, Montet-Abou K, Reynolds F, Weissleder R, Josephson L. Nanoparticle imaging of integrins on tumor cells. Neoplasia. 2006;8:214-222.

52. Bruns $\mathrm{O}$, Ittrich $\mathrm{H}$, Peldschus $\mathrm{K}$, et al. Real-time magnetic resonance imaging and quantification of lipoprotein metabolism in vivo using nanocrystals. Nat Nanotechnol. 2009;4:193-201.

53. Morawski A, Winter $\mathrm{P}, \mathrm{Yu} \mathrm{X}$, et al. Quantitative "magnetic resonance immunohistochemistry" with ligand-targeted ${ }^{19} \mathrm{~F}$ nanoparticles. Magn Reson Med. 2004;52:1255-1262.

54. Southworth R, Kaneda M, Chen J, et al. Renal vascular inflammation induced by Western diet in ApoE-null mice quantified by ${ }^{19} \mathrm{~F}$ NMR of VCAM-1 targeted nanobeacons. Nanomedicine. 2009;5:359-367.

55. Winter P, Morawski A, Caruthers S, et al. Molecular imaging of angiogenesis in early-stage atherosclerosis with alpha(v)beta3-integrin-targeted nanoparticles. Circulation. 2003;108:2270-2274.
56. Wickline S, Neubauer A, Winter P, Caruthers S, Lanza G. Applications of nanotechnology to atherosclerosis, thrombosis, and vascular biology. Arterioscler Thromb Vasc Biol. 2006;26:435-441.

57. Oostendorp M, Douma K, Hackeng T, et al. Quantitative molecular magnetic resonance imaging of tumor angiogenesis using cNGR-labeled paramagnetic quantum dots. Cancer Res. 2008;68:7676-7683.

58. Wang Y, de Rochefort L, Liu T, Kressler B. Magnetic source MRI: a new quantitative imaging of magnetic biomarkers. Conf Proc IEEE Eng Med Biol Soc. 2009;1:53-56.

59. Schabel M, Parker D. Uncertainty and bias in contrast concentration measurements using spoiled gradient echo pulse sequences. Phys Med Biol. 2008; 53:2345-2373.

60. Liu W, Dahnke H, Rahmer J, Jordan E, Frank J. Ultrashort T2* relaxometry for quantitation of highly concentrated superparamagnetic iron oxide (SPIO) nanoparticle labeled cells. Magn Reson Med. 2009;61:761-766.

61. Bowen C, Zhang X, Saab G, Gareau P, Rutt B. Application of the static dephasing regime theory to superparamagnetic iron-oxide loaded cells. Magn Reson Med. 2002;48:52-61.

62. de Lussanet Q, Langereis S, Beets-Tan R, et al. Dynamic contrast-enhanced MR imaging kinetic parameters and molecular weight of dendritic contrast agents in tumor angiogenesis in mice. Radiology. 2005;235:65-72.

63. Lu E, Wagner W, Schellenberger U, et al. Targeted in vivo labeling of receptors for vascular endothelial growth factor: approach to identification of ischemic tissue. Circulation. 2003;108:97-103.

64. Kenny L, Coombes R, Oulie I, et al. Phase I trial of the positron-emitting ArgGly-Asp (RGD) peptide radioligand ${ }^{18} \mathrm{~F}$-AH111585 in breast cancer patients. $J$ Nucl Med. 2008;49:879-886.

65. Morrison M, Ricketts S, Barnett J, Cuthbertson A, Tessier J, Wedge S. Use of a novel Arg-Gly-Asp radioligand, ${ }^{18} \mathrm{~F}$-AH111585, to determine changes in tumor vascularity after antitumor therapy. J Nucl Med. 2009;50:116-122.

66. Johnson L, Schofield L, Donahay T, Bouchard M, Poppas A, Haubner R. Radiolabeled arginine-glycine-aspartic acid peptides to image angiogenesis in swine model of hibernating myocardium. JACC Cardiovasc Imaging. 2008; 1:500-510.

67. Lee K, Jung K, Song S, et al. Radiolabeled RGD uptake and $\alpha_{\mathrm{v}}$ integrin expression is enhanced in ischemic murine hindlimbs. J Nucl Med. 2005;46:472478.

68. Indrevoll B, Kindberg G, Solbakken M, et al. NC-100717: a versatile RGD peptide scaffold for angiogenesis imaging. Bioorg Med Chem Lett. 2006;16: 6190-6193. 